\title{
Terapia Ocupacional y el menor de edad infractor
}

Ana María Gómez G.* Ana María Hernández R. **

Los niños y los jóvenes constituyen el futuro de la sociedad, asi de las oportunidades que tengan para satisfacer sus necesidades y desarrollar sus capacidades depende.0 la formación y adquisición de valores que permitan la organización de comunidades saludables. En la actualidad, los altos índices de marginalización, violencia y desprotección social han afectado, principalmente, las estructuras familiares, básicas en la construcción de la sociedad, vulnerando los derechos de la población infantil y juvenil, al limitar los escenarios de formación en el afecto, la alegría y la tolerancia.

El joven también se enfrenta a cambios físicos y emocionales que generan sentimiento de confusión en inconformismo, primando el deseo de imponer sus propias normas, influenciadas por los esquemas comportamentales, los arquetipos de héroes presentados en los medios de comunicación y en su propio contexto social.

Estos hechos influyen el surgimiento de subgrupos culturales, en los cuales los niños y los jóvenes buscan el reconocimiento y la satisfacción de sus necesidades, estableciendo normas de comportamiento, a menudo contrarias a las establecidas por la sociedad y la ley.

Frente a este panorama, el Estado asume la responsabilidad de hacer "Prevalecer los derechos de los niños y los jóvenes por encima de los demás"1, mediante la planeación e implementación de programas de Atención Integral al Joven, en

\footnotetext{
- Terapeuta Ocupacional: Lic. Pedagogía y Administración Educativa. Mag. Educación. Profesora Asistente, Universidad Nacional de Colombia.

- Terapeuta Ocupacional, Universidad Nacional de Colombia.

${ }^{1}$ CONSTITUCIÓN NACIONAL DE LA REPÚBLICA DE COLOMBIA. ARTÍCULO 44. 1991.
} 
los cuales se tiene en cuenta el respeto por sus derechos, la necesidad de establecer relaciones entre el joven, la familia, el sistema educativo y la comunidad, para favorecer el desarrollo de sus potenciales y de esta manera brindar oportunidades de ser el protagonista de su propia vida.La infracción juvenil se presenta como respuesta a la no satisfacción de necesidades básicas y el cumplimiento de determinadas normas para la aceptación en un subgrupo cultural (parche, pandilla); el volumen de menores en conflicto con la ley ha aumentado, siguiendo una tendencia similar a la delincuencia en el adulto.

\section{CARACTERÍSTICAS DEL MENOR INFRACTOR}

La información presentada a continuación se susténta en estudios realizados por la Fundación parà la Educación Superior FES (1999) y la Universidad externado de Colombia (1994, 1996), en los cuales se encuentra que la población de menores infractores se caracteriza por la incursión temprana en el delito (12 años) y lá posterior reincidencia en este. Los fenómenos de delincuencia juvenil se concentran en los centros urbanos y se especializan según la tendencia dominante en las diferentes regiones. La infracción juvenil se relaciona con la amplia red de actividades ilegales organizadas por los adultos, que vinculan al joven a diversos delitos como la prostitución y las organizaciones de producción, tráfico y consumo de Sustancias Psico-Activas (SPA). Esto ha generado el incremento en la gravedad de la infracción, presentándose acciónes en contra de la vida, la integridad personal y los atentados contra el patrimonio económico (robo), este último ocupa el primer lugar en cuanto a su frecuencia; debido a las condiciones de exclusión económica y laboral en las que crecen y se desenvuelven los menores, además de la adopción de valores y pautas sociales de consumo por fuera de las posibilidades de su comunidad. Además, se asocia a la infracción, el porte ilegal de armas, la posesión, tráfico y consumo de SPA y los atentados contra la libertad y el pudor sexual.

Teniendo en cuenta estos hechos, no se puede observar la infracción juvenil desde una perspectiva de acción individual, más bien debe ser entendida como un fenómeno que se construye socialmente, cuando en el menor se conjugan una serie de factores como' la exclusión familiar, social, escolar y laboral. En la historia de la mayoría de los menores infractores, se observa la ausencia del medio doméstico, escolar y comunitario adecuado; estos adolescentes han crecido en medios familiares y barriales agresivos, en donde han sido espectadores o víctimas de episo- 
dios violentos, los cuales los han marcado profundamente, llevándolos a entender que la violencia es una forma eficaz para resolver los conflictos.

La dinámica familiar se caracteriza por la ruptura o fragilidad de los roles familiares, afectivos y emocionales, lo cual limita la cohesión y la capacidad de satisfacer las necesidades básicas, emocionales y materiales. El conflicto se manifiesta en el abandono, maltrato, ausencia de la figura de autoridad (paterna), pautas de crianza ambiguas y modelos de comportamiento delincuencial. En cuanto a la exclusión escolar, se genera por las condiciones de pobreza que lleva a trabajar a padres e hijos para su mantenimiento básico, además se observa una limitación en las instituciones escolares, en cuanto a los recursos, para retener al estudiante y resolver los problemas de desarrollo social y comunitario, además de presentarse situaciones violentas al interior de estos centros.

En cuanto a la dinámica laboral, es notoria la dificultad de los jóvenes para acceder a trabajos dignos y bien remunerados, lo cual los lleva a la vagancia / desocupación productiva. Estos menores no tienen oportunidades de ingresar a medios laborales sanos y lícitos, por su baja escolaridad y capacitación, esto los coloca a merced de trabajos precarios, informales y temporales, que los ubica en la frontera difusa entre la informalidad, la ilegalidad y la delincuencia. La práctica cotidiana en este tipo de trabajos termina por desdibujar en el joven las fronteras de lo lícito e ilícito.

La deserción escolar y la vinculación a grupos callejeros indican el comienzo de la actividad delictiva, en la medida en que el joven aprende que la infracción posibilita el dinero fácil y rápido y lo coloca en una disposición negativa ante los trabajos rutinarios, lícitos y de poca remuneración; de la misma manera sucede al entrar en un sistema escolar. Además existe el problema del consumo de SPA, el $90 \%$ de los jóvenes infractores han manifestado el consumo de marihuana, bazuco, coca y pepas, entre otros, que hacen necesaria la implementación de políticas de prevención y rehabilitación.

\section{PROCESO DE ATENCIÓN JURÍDICA AL MENOR INFRACTOR}

En los procesos de atención jurídica, el menor de 18 años es reconocido como autor o participe de una infracción, pero se estima que no es responsable de sus 
actos, ha cometido un hecho o delito, pero no es culpable de ello, por eso se plantea la aplicación de Medidas en lugar de penas y sanciones; las medidas son procesos reeducativos planteados con el fin de proteger al menor y prevenir el delito; dichas medidas son determinadas por el juez, con el apoyo de un grupo interdisciplinario. Las medidas establecidas por la ley son*:

a. Amonestación: llamado de atención al menor y a las personas de quienes dependa.

b. Imposición de reglas de conducta: establecimiento de obligaciones y prohibiciones específicas.

c. Libertad asistida: el menor debe cumplir las reglas de conducta determinadas y asistir a centros con programas de reeducación a la población juvenil no institucionalizada.

d. Ubicación institucional: aplicada cuando no es conveniente que el menor permanezca en su medio familiar o social, por estar en gran amenaza, riesgo o violencia, y por la gravedad de la acción. Estas medidas se determinan de acuerdo con la naturaleza y las circunstancias de la infracción, teniendo en cuenta que los padres o acudientes son responsables de la situación del menor, lo cual le confiere un grado de Inimputabilidad, de tal manera que los procesos de reeducación / resocialización deben contemplar a la familia, la escuela y la comunidad como centros y facilitadores del cambio.

\section{PROCESO DE ATENCIÓN INTEGRAL AL MENOR INFRACTOR}

De acuerdo con el Código del Menor, el proceso de atención y determinación de la medida, así como los programas de intervención reeducativa, consta de las siguientes fases:

a. Recepción: en la cual el menor aprehendido es conducido a un centro especializado, en el cual estará a disposición del juez para el estudio y determinación de la medida pertinente.

\footnotetext{
• Confronte con: Código del Menor.
} 
b. Observación: considerada como una medida provisional, para el estudio y análisis de la situación personal, familiar y social del menor, con el fin de establecer un diagnóstico sobre la personalidad y la situación del mismo.

c. Tratamiento: que puede realizarse intra o extra institucionalmente, de acuerdo con la decisión del juez y el diagnóstico obtenido. Esta fase tiene por objeto la reeducación y/o resocialización del joven.

Durante el tratamiento el joven es confrontado con su realidad social y cultural, con el objetivo de promover cambios actitudinales que limiten las posibilidades de reincidir en la falta, además de fortalecer valores socialmente aceptados que permitan el desempeño lícito y productivo en su familia y sociedad. El proceso de intervención brinda al joven la oportunidad de reconocerse y reconocer su situación real, identificar sus intereses, motivaciones, posibilidades y oportunidades de ser un sujeto activo en el desarrollo de su vida, en la interacción con los demás y con su medio.

\subsection{Medios de intervención al menor infractor}

El proceso de atención integral debe ser enriquecido con medios y ambientes acordes a las necesidades de la población atendida. El ambiente se desarrolla en el marco de un trabajo interprofesional continuo, en el cual se conjuga el contexto formativo, terapéutico y lúdico'; se refiere a los espacios físicos y temporales en los cuales se llevan a cabo actividades académicas, técnicas y de socialización; en la intervención al menor en conflicto con la ley se incluye:

a. Ambiente formativo: consta de los procesos que fomentan la auto disciplina, la responsabilidad, el compromiso, la aceptación de reglas y normas de convivencia social y la introyección de límites.

b. Ambiente terapéutico: favorece el cumplimiento de los objetivos relacionados con la aceptación de la situación personal, familiar y social, para una posterior vida en comunidad, a través de las fases motivacional, relacional y psico-terapéutico.

\footnotetext{
${ }^{2}$ DIRECCIÓN NACIONAL DE ESTUPEFACIENTES (DNE). Proyecto pedagógico para la Atención Integral al menor infractor y contraventor de la Ley Penal Colombiana. Santa Fe de Bogotá, 1994.
} 
c. Ambiente lúdico: contempla espacios para el desarrollo personal mediante actividades deportivas, recreativas y de proyección artística, entre otras, para la exploración de aptitudes, habilidades, potencialidades y limitaciones. En estas actividades se incluyen los juegos de roles que llevan a la reflexión sobre la realidad personal y social, permiten el aprendizaje de normas, reglas y códigos de convivencia.

d. Ambiente evaluativo: el cual es constante en el proceso de atención, permitiendo la retroalimentación de las actividades y los medios utilizados mediante la evaluación y el seguimiento continuo y crítico.

Estos conceptos deben observarse en el desarrollo de programas de atención al menor en conflicto con la ley, en la búsqueda de la satisfacción de sus necesidades y la potencialización de sus capacidades.

\section{EL RETO DE TERAPIA OCUPACIONAL EN EL PROCESO DE REEDUCACIÓN DEL MENOR INFRACTOR}

La problemática del menor infractor es responsabilidad social, en la cual cada uno de los miembros de la sociedad esta llamado a aportar en la formación de los ciudadanos del mañana. Los Terapeutas ocupacionales, a partir de su formación, pueden apoyar el proceso de reeducación en los diferentes medios de intervención, partiendo de la caracterización ocupacional de la población, para establecer los criterios de acción terapéutica.

\subsection{Características de los menores infractores}

Los menores infractores, al igual que los adultos penalizados, representan una población marginada y estigmatizada que requiere de acciones concretas desde el marco legal y social, para prestar una atención integral, mediante programas reeducativos fundamentadas en el análisis critico de la historia personal, social y familiar del menor. Esta intervención debe basarse en los hallazgos a nivel de la ocupación, para facilitar los procesos que se encuentren deteriorados o en riesgo. Los menores infractores presentan características particulares en los 3 subsistemas de la ocupación humana; dichas características redundan en la capacidad de satisfacer sus necesidades básicas y potencializar sus capacidades. 
Cuadro 1. Características del menor infractor.

\begin{tabular}{|c|c|}
\hline SUBSISTEMA & CARACTERÍSTICAS \\
\hline \multirow[t]{3}{*}{ VOLICIÓN } & $\begin{array}{l}\text { Causación personal: dificultades para identificar las capacida- } \\
\text { des y habilidades personales, limitando el sentimiento de con- } \\
\text { trol de las acciones propias y la obtención de satisfacciones. }\end{array}$ \\
\hline & $\begin{array}{l}\text { Valores: sus convicciones personales están determinadas por } \\
\text { el grupo de amigos, negando en muchas ocasiones los valores } \\
\text { sociales que facilitan la convivencia. El control de las actitu- } \\
\text { des depende del mundo externo, no es percibido como una } \\
\text { responsabilidad personal. }\end{array}$ \\
\hline & $\begin{array}{l}\text { Intereses: búsqueda continua de actividades placenteras, del } \\
\text { agrado del grupo de amigos, subvalorando el trabajo produc- } \\
\text { tivo con metas a largo plazo. }\end{array}$ \\
\hline $\begin{array}{l}\text { HABITUACIÓN } \\
\text { Se presentan patrones } \\
\text { de comportamiento } \\
\text { orientados de acuerdo } \\
\text { con las normas del gru- } \\
\text { po de amigos (pandilla). }\end{array}$ & $\begin{array}{l}\text { Hábitos: dificultades para establecer y seguir rutinas en la eje- } \\
\text { cución de ocupaciones secuenciales, presentándose la inade- } \\
\text { cuada utilización del tiempo, el desequilibrio en la realización } \\
\text { de actividades de la Vida Diaria, la inadecuada presentación } \\
\text { personal y de sus trabajos, tanto escolares como laborales. }\end{array}$ \\
\hline HABITUACIÓN & $\begin{array}{l}\text { Roles: el sentido de identificación social esta determinado por } \\
\text { las normas del subgrupo, observándose dificultades en la con- } \\
\text { vivencia con otros y con el medio, el bajo sentido de pertenen- } \\
\text { cia y la aceptación de normas preestablecidas, desinterés por } \\
\text { asumir roles sociales, buscando seguir arquetipos de compor- } \\
\text { tamiento, no siempre adecuados. }\end{array}$ \\
\hline $\begin{array}{l}\text { EJECUCIÓN: } \\
\text { Conjunto de habilidades } \\
\text { necesarias para la reali- } \\
\text { zación de una tarea u } \\
\text { ocupación. }\end{array}$ & $\begin{array}{l}\text { Habilidades motoras: se observan patrones posturales inadecua- } \\
\text { dos que interfieren en la realización de tareas, siendo necesario } \\
\text { realizar ajustes posturales constantes. Las habilidades motoras } \\
\text { finas se encuentran alteradas, presentándose torpeza en la rea- } \\
\text { lización de acabados y la ejecución tareas de destreza. La } \\
\text { marcha se caracteriza por la presencia de modismos. Los } \\
\text { menores infractores presentan descargas motoras constantes, } \\
\text { manifiestas en inquietud, inadecuado manejo espacial y mo- } \\
\text { vimientos bruscos. }\end{array}$ \\
\hline
\end{tabular}




\begin{tabular}{|l|l|}
\hline SUBSISTEMA & \multicolumn{1}{c|}{ CARACTERÍSTICAS } \\
\hline & $\begin{array}{l}\text { Habilidades del desempeño: se presentan dificultades en la } \\
\text { atención y la permanencia en las tareas complejas, limitando } \\
\text { la finalización de tareas secuenciales. De la misma manera la } \\
\text { planeación de actividades y los procesos de toma de decisio- } \\
\text { nesy resolución de conflictos se muestran limitados, requirien- } \\
\text { do de asistencia constante. }\end{array}$ \\
\hline & $\begin{array}{l}\text { Habilidades de comunicación e interacción: los jóvenes en con- } \\
\text { flicto con la ley adoptan expresiones características, denomi- } \\
\text { nadas jergas, que buscan consolidar el grupo social y excluir a } \\
\text { las personas que no consideran aptas. El menor infractor tiene } \\
\text { dificultades para establecer compromisos e intercambiar infor- } \\
\text { mación. }\end{array}$ \\
\hline
\end{tabular}

Elaborado por Gómez A. Hernández A. 2001.

A pesar de las dificultades que presenta el menor infractor, se encuentra un joven con capacidades que no han podido desarrollarse en forma adecuada para si mismo y la sociedad, por la carencia de espacios para la expresión de sus sentimientos positivos, el afecto, los valores y la solidaridad.

De acuerdo con estas características, el reto del terapeuta ocupacional está encaminado al fortalecimiento de valores y normas sociales adecuadas, que permitan la satisfacción de las necesidades básicas y la potencialización de las capacidades, con el fin de lograr la construcción de comunidades saludables y productivas que prevengan la aparición y reincidencia de conductas delictivas; haciendo uso de los mecanismos establecidos por la ley.

\section{INTERVENCIÓN DE TERAPIA OCUPACIONAL}

De esta manera, la atención al menor infractor debe ser un trabajo interdisciplinario, a nivel intra $e$ interinstitucional y comunitario para garantizar la efectividad del proceso de atención integral, el cual está inmerso en un ambiente formativo, terapéutico y lúdico que promueva la autodisciplina, la percepción de habilidades, la capacitación en un oficio o labor digna que permita el conocimiento de las capacidades personales y la posterior ubicación social, escolar y laboral satisfactoria. 
El terapeuta ocupacional está llamado a enriquecer el proceso de reeducación a través de asesorías y evaluación e implementación de programas, teniendo en cuenta la problemática ocupacional del menor y las circunstancias en las cuales se desenvuelve; a partir de la orientación prevocacional y vocacional, la nivelación escolar y laboral, la adquisición y recuperación de hábitos acordes a las necesidades e intereses de la población atendida.

De acuerdo con los parámetros establecidos por la Ley, la atención al menor infractor se realiza en tres fases (Ver Diagrama 1):

Diagrama 1. Proceso de atención al menor infractor.

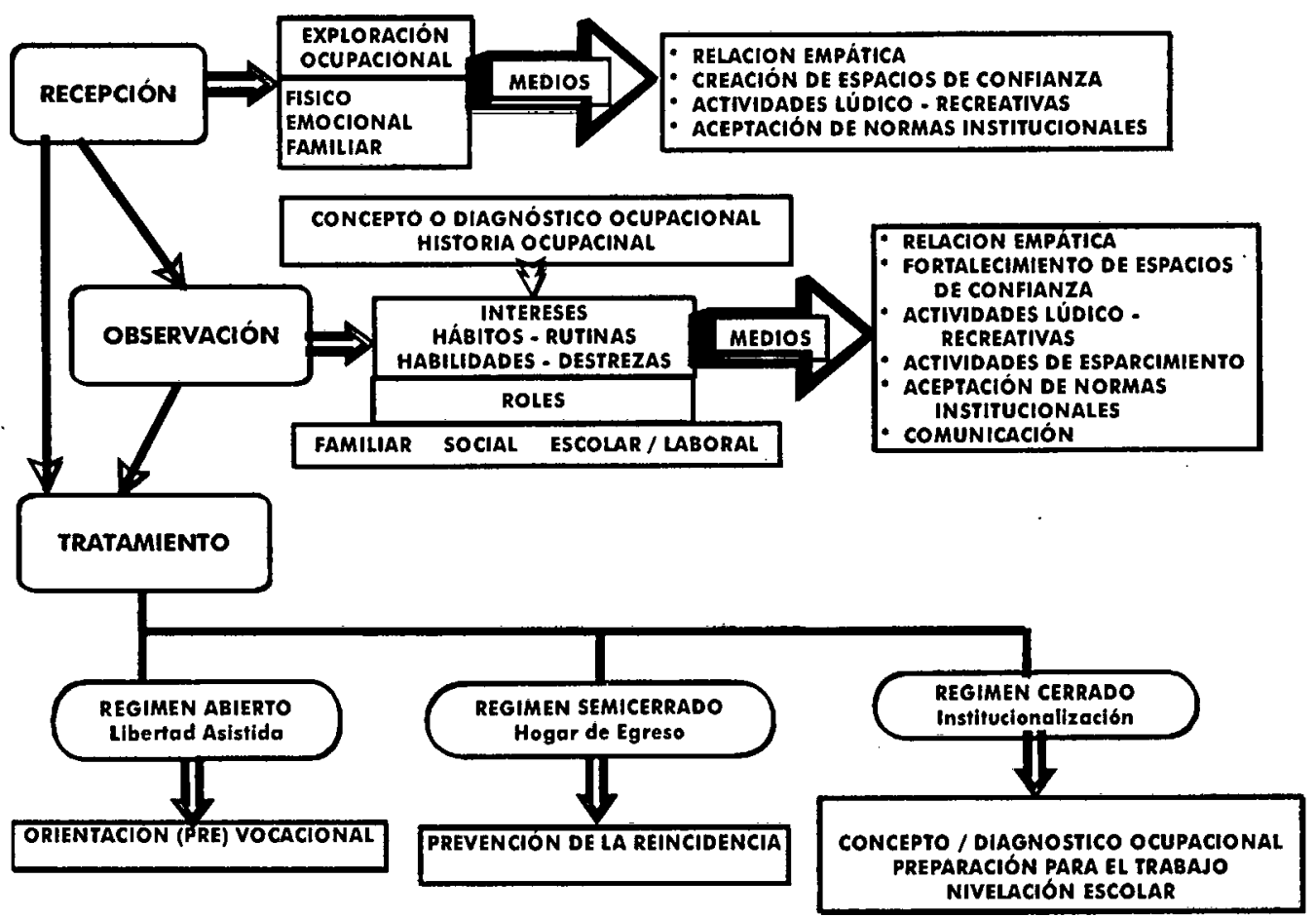

Elaborado por Gómez, Ana María; Hernández, Anamaría, 2001.

a. La recepción (5 días): en este periodo, por el tiempo disponible, el terapeuta ocupacional realiza un primer acercamiento al joven, y se inicia la explora- 
ción ocupacional, mediante la observación del comportamiento del menor en la institución, la realización de actividades lúdicas, la creación de espacios de confianza, y la observación del comportamiento del menor en la institución.

b. La observación (60 días): es el segundo momento en la intervención al menor infractor, durante esta etapa se debe iniciar la historia ocupacional y el establecimiento de un concepto ocupacional, enfatizando en los intereses del joven, para plantear actividades que faciliten la satisfacción de necesidades y se brinden oportunidades para la potencialización de capacidades. Los medios empleados en esta etapa incluyen la entrevista, las aplicación de evaluaciones, la observación del comportamientos, la realización de actividades lúdicas-recreativas, actividades con propósito, el énfasis en la adaptación al ambiente, el seguimiento y aceptación de normas y rutinas institucionales. Es importante que se fortalezcan los espacios de confianza mediante una adecuada relación empática, en la cual se permita la observación del nivel de interiorización de los roles sociales, familiares y escolares/laborales, así como de los intereses y las expectativas del menor una vez retorne a su ambiente cotidiano.

c. Por último el tratamiento (6 meses - 3 años): En esta fase se realiza una intervención más profunda, en la cual se enfatiza en los procesos de orientación prevocacional-vocacional y en las actividades de reeducación que facilitan la reincorporación del menor a su ambiente social y se previene la reincidencia en la infracción; por lo tanto es necesario incluir a la familia, la escuela y la comunidad en los procesos de atención. Dichos procesos se presentan de acuerdo con el tipo de medida determinada. (Ver Diagrama 2).

Régimen abierto (Libertad Asistida): se refiere a programas realizados en instituciones en las cuales el joven se encuentra en su ambiente cotidiano y asiste a una institución con programas de reeducación. En estas instituciones se realizan programas de orientación prevocacional y vocacional, enfatizando en el fortalecimiento de los valores sociales que facilitan la vida comunitaria de manera lícita y productiva; se realizan talleres para el adecuado manejo del tiempo libre y de las relaciones interpersonales, con el fin de canalizar las capacidades de los jóvenes hacia el desarrollo de conductas saludables. El programa de orientación vocacional debe realizarse en tres fases, en las cuales el menor en conflicto con la ley reconoce sus intereses y sus capacidades, para realizar una selección ocupacional satisfactoria. 
Diagrama 2. Proceso de atención del menor en tratamiento.

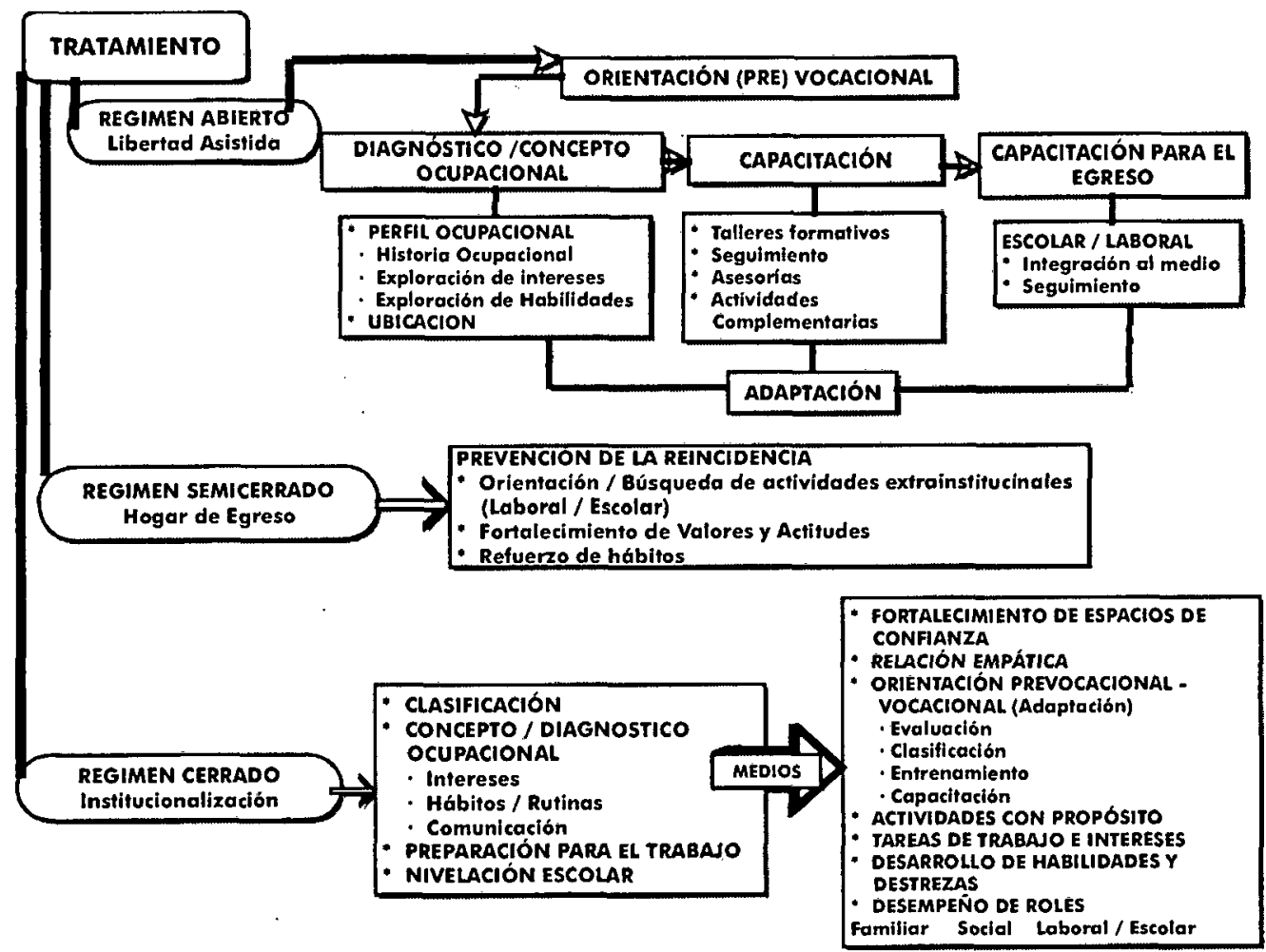

Elaborado por: Gómez, Ana Maria; Hernández, Anamaría. 2000.

En la primera fase, el terapeuta debe identificar los intereses, en una segunda fase se reconocerán las capacidades y en la tercera se realiza la elección ocupacional y se llevan a cabo los procesos de capacitación y adiestramiento. La mayor dificultad de estos programas reside en la baja constancia de los usuarios y la falta de compromiso de las familias y comunidades; su mayor fortaleza se centra en la posibilidad de aplicar los conceptos al medio cotidiano y retroalimentar los procesos reeduacativos de acuerdo con las respuestas de los jóvenes y del ambiente.

Régimen semicerrado (Instituciones de Egreso): En estas instituciones los jóvenes interactúan con el ambiente social externo, pero deben permanecer en una institución, debido a los riesgos que representa el medio cotidiano. En estas instituciones se realizan programas de prevención de la reincidencia, orientación para la 
búsqueda de actividades extrainstitucionales, bien sean de tipo escolar o laboral; las actividades realizadas enfatizan en el reconocimiento y apropiación de valores y actitudes tendientes a favorecer un estilo de vida saludable, además del refuerzo de hábitos, el manejo del dinero, el manejo del tiempo y la sana diversión.

Régimen cerrado (Institucionalización): En este régimen se encuentran incluidos los jóvenes que por sus condiciones sociales o por la gravedad de su falta, requieren de mayor asistencia en protección y prevención de la reincidencia. En estas instituciones es necesario realizar una clasificación de los jóvenes, de acuerdo con el diagnóstico ocupacional establecido. Los programas ofrecidos incluyen la preparación para el trabajo, la nivelación escolar, la identificación de intereses, hábitos, rutinas, niveles de comunicación.

Los medios empleados incluyen la orientación prevocacional y vocacional, a partir de la evaluación, el entrenamiento y la capacitación, la realización de actividades con propósito, el refuerzo de los espacios de confianza, las actividades que fomentan el desarrollo de habilidades y destrezas. En el régimen cerrado se debe tener en cuenta la inclusión del ambiente del menor, su realidad histórica, social y cultural, de manera que se faciliten la continuidad y mantenimiento de los procesos reeducativos en la vida del joven una vez recupera su libertad, por lo tanto es útil establecer programas de seguimiento en casa.

La orientación prevocacional y vocacional, busca que el joven realice una selección ocupacional (escolar o laboral) de acuerdo con sus intereses y su experiencia. En el caso de los menores infractores de la ley penal, en proceso de reeducación, la orientación prevocacional y vocacional, está dirigida hacia la promoción de aprendizajes sanos que fomenten el desarrollo ý reconocimiento de las habilidades propias, el replanteamiento de los valores e intereses y la identificación de dificultades frente a la elección de una ocupación digna y lícita.

El proceso de orientación ha de permitir la interpretación de valores personales y sociales con respecto al trabajo honesto y productivo, además de afianzar los principios de respeto por sí mismo y por los demás, las costumbres y rutinas de trabajo, autocuidado y esparcimiento. Es responsabilidad del terapeuta ocupacional, apoyar al menor en el proceso de reconocimiento y aceptación de sí mismo, así como el logro de un posicionamiento en su grupo social, a través de la ocupación, con la plena realización de sus necesidades de Ser - Tener - Hacer - Estar. 


\section{CONCLUSIONES Y RECOMENDACIONES}

El trabajo dentro de la reeducación / resocialización del menor en conflicto con la Ley Penal es visto desde la orientación vocacional en una doble perspectiva: como medio Terapéutico/ educativo y como finalidad, donde la educación desarrolla las capacidades ocupacionales y el valor terapéutico en que el trabajo modifique el interior del hombre para su bienestar personal y social.

El rol del Terapeuta ocupacional en la Orientación (pre)vocacional, se dirige a la promoción de aprendizajes que contribuyan en la prevención de trastornos y fomenten la calidad de vida en el entomo educativo y ocupacional / laboral. El terapeuta ocupacional, acompaña al joven en el desarrollo de sus habilidades, el aclarar sus intereses y dificultades frente a una elección, en general lo ayuda a conocerse.

Mediante el desempeño del terapeuta ocupacional en programas de orientación vocacional se busca que el joven perciba este proceso como un proceso de cambio a través de la ocupación, como fuente de entrenamiento de habilidades, desarrollo de cualidades humanas. El joven aprende a valorar el rol escolar / laboral como una "Obra bien hecha" que abre el camino a la responsabilidad individual y social; esto influye en la calidad técnica, el valor utilitario, los conocimientos y el cultivo de la producción intelectual y material, así el joven recrea su mundo de posibilidades para una mejor calidad de vida.

En cuanto al papel de la Academia en la atención al menor infractor, se parte de la investigación, la docencia y la extensión como procesos interrelacionados que definen el que hacer de la educación superior, cuya responsabilidad social es contribuir desde el escenario académico a la comprensión de los procesos poblacionales del país, y plantear alternativas de solución.

Como lo expresó Alejo ${ }^{3}$, en el contexto universitario el diseño, desarrollo y evaluación de programas de extensión en el campo de la delincuencia, toma especial importancia, puesto que a partir de la praxis se puede generar conocimiento; los

\footnotetext{
${ }^{3}$ Alejo, Henry. "El papel de la Educación Superior en la Prevención y Resocialización del Infractor". En: Memorias del Primer Congreso Internacional sobre la Prevención y Resocialización del Infractor Penal. Universidad Católica de Colombia. Instituto Colombiano de Bienestar Familiar (ICBF), Instituto Nacional Penitenciario y Carcelario (INPEC), Clínica Toxicológica. Santa Fe de Bogotá, 1993. p.220.
} 
programas de extensión brindan a docentes y alumnos posibilidades valiosas para su formación, en los cuales se contrastan diversos marcos de referencia teóricos, éticos y metodológicos con la realidad social. Desde el campo de acción de Terapia ocupacional, se han realizado investigaciones y propuestas de intervención a la población de menores en riesgo y menores infractores, identificando la importancia del ambiente físico, social y familiar en los procesos de prevención y reeducación a esta población.

\section{REFERENCIAS BIBLIOGRÁFICAS}

Alejo, Henry. "El papel de la Educación Superior en la Prevención y Resocialización del Infractor". En: Memorias del Primer Congreso Internacional sobre la Prevención y Resocialización del Infractor Penal. Universidad Católica de Colombia. Instituto Colombiano de Bienestar Familiar (ICBF), Instituto Nacional Penitenciario y Carcelario (INPEC), Clínica Toxicológica, Santa Fe de Bogotá, 1993.

Casas P, Diana Patricia y González P, Gilma Inés. Orientación vocacional para menores infractores que se encuentran en una institución de reeducación con medida de Libertad Asistida en Soacha. Santa Fe de Bogotá, 1999. Trabajo de Grado (Terapia Ocupacional). Universidad Nacional de Colombia.

CENTRO DE INVESTIGACIONES SOBRE DINÁMICA SOCIAL. Tendencias en la infracción y contravención entre menores y Ponderación de la calidad de la respuesta institucional. Universidad Externado de Colombia. Santa Fe de Bogotá, Colombia, 1997.

DIRECCIÓN NACIONAL DE ESTUPEFACIENTES (DNE). INSTITUTO COLOMBIANO DE BIENESTAR FAMILIAR (ICBF) y PROGRAMA DE LAS NACIONES UNIDAS PARA EL CONTROL Y FISCALIZACIÓN DE DROGAS (UNDCP). Proyecto pedagógico para la atención integral al menor infractor y contraventor de la Ley Penal Colombiana. Santa Fe de Bogotá, 1994.

Menores infractores y contraventores en el departamento de Santander. Diseño del Proyecto de Atención Integral. Bucaramanga, julio de 1995.

FUNDACIÓN PARA LA EDUCACIÓN SUPERIOR. Tres estudios inéditos sobre los Menores Infractores en Colombia. Santa Fe de Bogotá, 1992.

Gómez, Ana María. "Educación y trabajo en las cárceles". En: Revista Ocupación Humana. Volumen 6, No. 3, p. 48. Santa Fe de Bogotá, 1996. 
INSTITUTO COLOMBIANO DE BIENESTAR FAMILIAR (ICBF). El menor infractor y contraventor, plan operativo, creación, organización y funcionamiento de las instituciones y servicios. Santa Fe de Bogotá, 1992.

Kielhofner, Gary. El Modelo de la Ocupación Humana. Memorias del VIII Congreso Nacional de Terapia Ocupacional. Santa Fe de Bogotá, 1992.

MINISTERIO DE JUSTICIA. Código del menor.

Sánchez, Rosa; Sandoval, Mariela y Vargas, Aracely. Diferencias a nivel de encausamiento personal y valores entre adolescentes no infractores y menores infractores. Trabajo de Grado (Terapia Ocupacional). Universidad Nacional de Colombia, 1992.

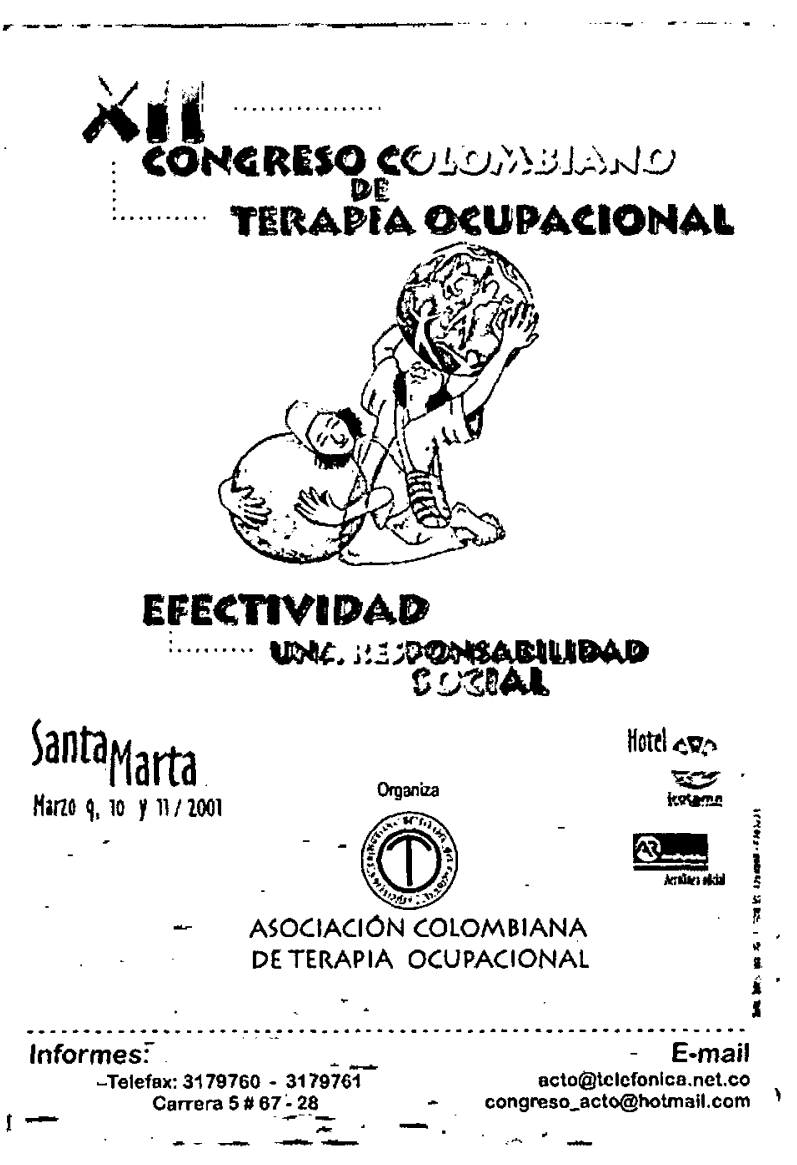

